\title{
Dynamical Analysis and Parameter Estimation of a Model of Hepatitis B Disease Spread in Malang
}

\author{
Fitroh Aulani ${ }^{{ }^{*}}$, Wuryansari Muharini Kusumawinahyu², Isnani Darti ${ }^{2}$ \\ ${ }^{1}$ Master Program of Mathematics, Faculty of Mathematics and Natural Sciences, University of Brawijaya, Malang, \\ Indonesia \\ ${ }^{2}$ Department of Mathematics, Faculty of Mathematics and Natural Sciences, University of Brawijaya, Malang, Indonesia
}

\section{Abstract}

In this article, a model representing the spread of Hepatitis B disease is constructed as a nonlinear autonomous system. The model divides the considered human population into three classes, namely susceptible, infected, and recovered class. The dynamical analysis shows that there are two equilibrium points in the model, namely a disease-free equilibrium point and an endemic equilibrium point. The existence and stability of the equilibrium points depend on the basic reproduction number $\left(\mathcal{R}_{0}\right)$. The disease-free equilibrium point is local asymptotically stable when $\mathcal{R}_{0}<1$, while the endemic equilibrium point exists and is local asymptotically stable if $\mathcal{R}_{0}>1$. The five parameters of the model are estimated by applying Downhill Simplex (Nelder-Mead) Algorithm and by using the infected data cases taken from such a hospital in Malang. The estimated parameters are the transmission of infection rate, the saturation rate, the vaccination rate, the recovery rate, and the immunity loss rate. The resulting parameter estimation supports the analytical result and is used to illustrate the analytical results numerically. Based on the considered model and the result of the parameters estimation, it can be concluded that the Hepatitis B spread in Malang is controllable.

Keywords: downhill simplex (Nelder-Mead) algorithm, dynamical analysis, hepatitis B model, parameter estimation.

\section{INTRODUCTION}

Hepatitis $B$ disease is a type of infectious disease caused by the Hepatitis B Virus (HBV). That virus is a type of the virus family Hepadnaviridae [1]. HBV is more easily spread than other viruses, like Hepatitis C Virus (HCV) or Human Immunodeficiency Virus (HIV). HBV can also be transmitted through sexual transmission [2]. According to a nationwide report in 2007 and 2013, Hepatitis B cases in Indonesia are indicated from high to moderate [3].

Many types of research have been conducted to describe the epidemic phenomenon by constructing mathematical models which divide the population into some classes. The classic epidemic model called the SIR model considers three classes, namely Susceptible, Infected, and Recovery class. The SIR model is introduced in 1927, and the model is governed by the bilinear incidence rate [4]. Many modifications are applied to the classic SIR model based on the disease character. Capasso and Serio [5] in 1978 modified the SIR model by applying a saturated incidence rate. In 2019, Khan et al. [6] constructed a SIR model Hepatitis B with a saturated transmission rate and added the vaccination factor to the model. Furthermore,

\footnotetext{
Correspondence address:

Fitroh Aulani

Email : fitrohaulani96@gmail.com

Address : Dept. Mathematics, University of Brawijaya, Veteran Malang, 65145
}

Khan et al. also assumed that recovered individuals have permanent immunity.

Generally, the mathematical models contain some parameters that can be used to predict the behavior of the disease spread as long as their values are known. To determine the parameter values, we can do the estimation process to approximate the actual data with the solution of the model. Golgeli [7] analyzed a SIR Hepatitis B model with a bilinear transmission rate and performed the parameter estimation using a data set taken in Turkey. This model consists of four parameters, and one of the parameters is estimated. Golgeli concluded that Hepatitis B in Turkey stabilizes into a disease-free state.

Based on the previous research, in this paper, a Hepatitis B disease model was constructed by modifying the model of Khan et al. [6] into the SIRS model. A dynamical analysis is conducted by determining the equilibrium point and the local stability of the equilibrium point. The parameter values of the SIRS model are obtained by employing the parameter estimation process. The infected cases data of Hepatitis B was retrieved from Dr. Saiful Anwar Hospital, a governmental hospital in Malang. Data from January 2015 to December 2019 were used in the estimation process. Furthermore, numerical simulation was performed to illustrate the analytical results related to the spread of Hepatitis B in Malang. 


\section{MATERIAL AND METHOD \\ Model Formulation}

In this research, a Hepatitis B disease model was reconstructed and represented as a SIRS model by assuming that the recovered individuals can lose their immunity. Hence the recovered individual can be reinfected. The incidence rate is also modified into a saturated incidence rate. Based on those modifications, a nonlinear autonomous system consisting of three variables and eight parameters is proposed to describe the Hepatitis B spread.

\section{Equilibrium Point and Local Stability}

The equilibrium point of the model is obtained when the size of all class of population in the model are constant. The linearization process around the equilibrium point is carried out to analyze the local stability of the equilibrium point. The eigen values of the Jacobian matrix coming from the linearization process provides the information about the stability of the equilibrium point. If all of the eigen values of Jacobian matrix have negative real part, the equilibrium point is local asymptotically stable.

\section{Parameter Estimation}

The estimation process determines the value of the parameter in the model by minimizing the difference between the estimated solution of the model and the real data iteratively. Hence, the first step of the estimation process is considering the objective function which is used in [8]:

$$
O_{f}=\sum_{1}^{i} R_{i}^{2}=\sum_{1}^{i}\left(Y_{i}-f\left(x_{i}, \theta\right)\right)^{2}
$$

where $R_{i}$ is the $\mathrm{i}^{\text {th }}$ residual, $Y_{i}$ is the $\mathrm{i}^{\text {th }}$ data, and $f\left(x_{i}, \theta\right)$ is the numerical solution of the model with the vector of the parameters $\theta$. The objective function is minimized by using the Nelder-Mead Algorithm [9,10], as follows.

1. Choose the initial value of the parameter $\theta$ and the initial value of population as the variable in the model.

2. Generate the population of the model $f\left(x_{i}, \theta\right)$ using the Runge-Kutta $4^{\text {th }}$ order method.

3. Determine the objective function (1) and sorted the values of objective function from smallest to largest values

$O_{f}\left(\vec{\theta}_{1}\right) \leq O_{f}\left(\vec{\theta}_{2}\right) \leq O_{f}\left(\vec{\theta}_{3}\right) \leq \cdots \leq O_{f}\left(\vec{\theta}_{i+1}\right)$.
4. Calculate the reflection point

$$
\vec{\theta}_{r}=\overrightarrow{\bar{\theta}}+\rho\left(\overrightarrow{\bar{\theta}}-\overrightarrow{\bar{\theta}}_{i+1}\right)
$$

where $\overrightarrow{\bar{\theta}}=\sum_{1}^{i} \frac{\overrightarrow{\theta_{l}}}{l}$. The standard value for the coefficient of reflection is $\rho=1$.

5. If $O_{f_{1}} \leq O_{f_{r}}<O_{f_{i}}$, the reflection point is accepted. Terminate the iteration.

6. If $O_{f_{r}}<O_{f_{1}}$, calculate the expansion point

$$
\vec{\theta}_{e}=\overrightarrow{\vec{\theta}}+\chi\left(\vec{\theta}_{r}-\overrightarrow{\vec{\theta}}\right) .
$$

The standard value for the coefficient of expansion is $\chi=2$. If $O_{f_{e}}<O_{f_{r}}$, accepted the expansion point $\vec{\theta}_{e}$ and terminated the iteration. If $O_{f_{e}} \geq O_{f_{r}}$, accepted the reflection point $\vec{\theta}_{r}$ and terminated the iteration.

7. Determination of the contraction point.

a. Outside. If $O_{f_{i}} \leq O_{f_{r}}<O_{f_{i+1}}$ calculate the outside contraction point

$$
\vec{\theta}_{c}=\overrightarrow{\bar{\theta}}+\hat{\gamma}\left(\vec{\theta}_{r}-\overrightarrow{\bar{\theta}}\right) \text {. }
$$

The standard value for the coefficient of contraction is $\hat{\gamma}=\frac{1}{2}$. If $O_{f_{c}} \leq O_{f_{r}}, \vec{\theta}_{c}$ is accepted and terminated the iteration otherwise go to the next step.

b. Inside. If $O_{f_{r}} \geq O_{f_{i+1}}$ calculate the inside contraction point

$$
\vec{\theta}_{c c}=\overrightarrow{\vec{\theta}}-\hat{\gamma}\left(\overrightarrow{\vec{\theta}}-\vec{\theta}_{i+1}\right) .
$$

If $O_{f_{c c}} \leq O_{f_{i+1}}, \vec{\theta}_{c c}$ is accepted and terminated the iteration otherwise go to the next step.

8. Shrink (reduction). Evaluate the objective function $O_{f}$ at each point $\vec{v}_{k}$, which the point is obtained based on

$$
\vec{v}_{k}=\vec{\theta}_{1}+\sigma\left(\vec{\theta}_{k}-\vec{\theta}_{1}\right)
$$

where $k=2, \ldots, i+1$. Replace the $\vec{\theta}_{k}$ point with $\vec{v}_{k}$. The next iteration consist of $\vec{\theta}_{1}, \vec{v}_{2}, \ldots, \vec{v}_{i+1}$. The standard value for the coefficients of shrink (reduction) is $\sigma=\frac{1}{2}$.

9. The iteration ends if the tolerance criteria have been reached as [11].

$$
\left\|\vec{\theta}_{i+1}-\vec{\theta}_{i}\right\| \leq \epsilon_{\theta} \text { and } O_{f}\left(\vec{\theta}_{i}\right)-O_{f}\left(\vec{\theta}_{i+1}\right) \leq \epsilon_{f} \text {. }
$$

\section{Numerical Simulation}

The parameter values obtained from the parameter estimation process are used in this part. The model is simulated numerically by using Runge-Kutta $4^{\text {th }}$ order method to confirm the analytical results and to show the dynamic of the model. 


\section{Data Collection}

This research uses secondary data of the number of infected Hepatitis B from January 2015 to December 2019. The data was taken from RSUD Dr. Saiful Anwar Malang. The data of the infected individuals are presented at Table 1.

Table 1. The number of HBV infected individuals

\begin{tabular}{lccccc}
\hline & \multicolumn{5}{c}{ Number of infected } \\
\cline { 2 - 6 } \multicolumn{1}{c}{ Times } & $\mathbf{2 0 1 5}$ & $\mathbf{2 0 1 6}$ & $\mathbf{2 0 1 7}$ & $\mathbf{2 0 1 8}$ & $\mathbf{2 0 1 9}$ \\
\hline January & 27 & 6 & 12 & 14 & 7 \\
February & 13 & 5 & 6 & 14 & 7 \\
March & 14 & 2 & 10 & 6 & 7 \\
April & 8 & 5 & 8 & 11 & 11 \\
May & 4 & 4 & 9 & 12 & 16 \\
June & 5 & 8 & 5 & 2 & 7 \\
July & 2 & 8 & 10 & 12 & 14 \\
August & 13 & 7 & 15 & 7 & 9 \\
September & 3 & 3 & 18 & 7 & 12 \\
October & 4 & 12 & 16 & 13 & 15 \\
November & 6 & 10 & 18 & 13 & 11 \\
December & 6 & 11 & 10 & 11 & 10 \\
\hline
\end{tabular}

\section{RESULT AND DISCUSSION}

Model Formulation

Based on the assumption and the modification on the model of Khan et al. in 2019 [6] that have been described before, we propose a model of Hepatitis B transmission as follows.

$$
\begin{aligned}
& \frac{d S(t)}{d t}=\Lambda-\frac{\alpha S(t) I(t)}{1+\gamma I(t)}-\left(\mu_{0}+v\right) S+\delta R(t) \\
& \frac{d I(t)}{d t}=\frac{\alpha S(t) I(t)}{1+\gamma I(t)}-\left(\mu_{0}+\mu_{1}+\beta\right) I(t) \\
& \frac{d R(t)}{d t}=\beta I(t)+v S(t)-\left(\mu_{0}+\delta\right) R(t)
\end{aligned}
$$

where $S(t), I(t)$, and $R(t)$ represent the susceptible individual, the infected individual, and the recovered individual at time $t$, respectively. The parameters $\Lambda, \alpha, \mu_{0}, \mu_{1}, \gamma, v, \beta$, and $\delta$ represent the birth rate, the transmission of infection rate, the natural mortality rate, the death rate because of Hepatitis $B$ infection, the saturation rate, the vaccination rate, the recovery rate, and the immunity loss rate respectively. The density of the total population considered in the model is the sum of the density of susceptible, infected, and recovered classes, namely $N(t)=$ $S(t)+I(t)+R(t)$.

\section{Equilibrium Point of Model}

Since the equilibrium point of the model (2) is obtained when the size of all class of population in the model are constant, then it is reached when $\frac{d S}{d t}=\frac{d I}{d t}=\frac{d R}{d t}=0$. It can be proved easily, that there are two equilibrium points, namely the disease-free equilibrium point

$$
E_{0}=\left(S_{0}, I_{0}, R_{0}\right)=\frac{\Lambda\left(\mu_{0}+\delta\right)}{\phi}, 0, \frac{v \Lambda}{\phi}
$$

and the endemic equilibrium point $E^{*}=$ $\left(S^{*}, I^{*}, R^{*}\right)$

$$
\begin{gathered}
S^{*}=\frac{\eta\left(1+\gamma I^{*}\right)}{\alpha}, \\
I^{*}=\frac{\Lambda \alpha\left(\mu_{0}+\delta\right)-\eta \phi}{\eta\left(\alpha \mu_{0}+\gamma \phi\right)+\alpha \delta\left(\mu_{0}+\mu_{1}\right)}, \\
R^{*}=\frac{\beta I^{*}+v S^{*}}{\mu_{0}+\delta} .
\end{gathered}
$$

Here $\eta=\mu_{0}+\mu_{1}+\beta$ and $\phi=\mu_{0}\left(\mu_{0}+\delta+v\right)$. Furthermore, by applying the Next Generation Matrix method [12], the basic reproduction number $\left(\mathcal{R}_{0}\right)$ is easily formulated, namely

$$
\mathcal{R}_{0}=\frac{\alpha \Lambda\left(\mu_{0}+\delta\right)}{\eta \phi} .
$$

It is clear that the disease-free equilibrium always exists, while the endemic equilibrium exists when $\mathcal{R}_{0}>1$.

\section{Stability of the Equilibrium Point}

The local stability of the equilibrium point can be determined through the linearization process, which provide the Jacobian matrix

$$
J=\left(\begin{array}{ccc}
-\frac{\alpha I}{1+\gamma I}-\mu_{0}-v & -\frac{\alpha S}{(1+\gamma I)^{2}} & \delta \\
\frac{\alpha I}{1+\gamma I} & \frac{\alpha S}{(1+\gamma I)^{2}}-\eta & 0 \\
v & \beta & -\mu_{0}-\delta
\end{array}\right) .
$$

The result of the stability analysis is presented in the following theorems.

Theorem 1. if the basic reproduction number is less than one $\left(\mathcal{R}_{0}<1\right)$, the disease-free equilibrium point $\left(E_{0}\right)$ of system (2) is local asymptotically stable.

Proof. The Jacobian matrix at $E_{0}$ is

$$
J\left(E_{0}\right)=\left(\begin{array}{ccc}
-\mu_{0}-v & -\frac{\alpha \Lambda\left(\mu_{0}+\delta\right)}{\phi} & \delta \\
0 & \frac{\alpha \Lambda\left(\mu_{0}+\delta\right)}{\phi}-\eta & 0 \\
v & \beta & -\mu_{0}-\delta
\end{array}\right) .
$$

$J\left(E_{0}\right)$ has three eigenvalues, namely $\lambda_{1}=$ $-\left(\mu_{0}+\delta+v\right), \lambda_{2}=-\mu_{0}$ and $\lambda_{3}=\eta\left(\mathcal{R}_{0}-1\right)$. All eigenvalues are negative if $\mathcal{R}_{0}<1$. Hence, the disease-free equilibrium $E_{0}$ is local asymptotically stable when $\mathcal{R}_{0}<1$. 
Theorem 2. If $\mathcal{R}_{0}>1$, the endemic equilibrium point $\left(E^{*}\right)$ of system (2) exist and is local asymptotically stable.

Proof. The Jacobian matrix at $E^{*}$ is

$$
J\left(E^{*}\right)=\left(\begin{array}{ccc}
-\frac{\alpha I^{*}}{\omega_{1}}-\omega_{2} & -\frac{\alpha S^{*}}{\omega_{1}^{2}} & \delta \\
\frac{\alpha I^{*}}{\omega_{1}} & \frac{\alpha S^{*}}{\omega_{1}^{2}}-\eta & 0 \\
v & \beta & -\omega_{3}
\end{array}\right)
$$

where

$$
\omega_{1}=1+\gamma I^{*}, \omega_{2}=\mu_{0}+v, \omega_{3}=\mu_{0}+\delta
$$

Based on calculated $\left|J\left(E^{*}\right)-\lambda I\right|$, we get a polynomial equation of third degree.

where

$$
\lambda^{3}+a_{1} \lambda^{2}+a_{2} \lambda+a_{3}=0,
$$

$a_{1}=\omega_{2}+\omega_{3}+\frac{I^{*}}{\omega_{1}}(\alpha+\eta \gamma)$

$a_{2}=\frac{I^{*}}{\omega_{1}}\left(\alpha \omega_{3}+\alpha \eta+\eta \gamma \omega_{3}+\eta \gamma \omega_{2}\right)+\phi$,

$a_{3}=\frac{I^{*}}{\omega_{1}}\left(\alpha \mu_{0} \eta+\alpha \delta \mu_{0}+\alpha \delta \mu_{1}+\phi \eta \gamma\right)$.

Routh-Hurwitz criterion stated that all of the root of the equation (3) have negative real part if $a_{1}>0, \quad a_{3}>0, \quad$ and $a_{1} a_{2}-a_{3}>0$ [13]. It is clear that $a_{1}>0$ and $a_{3}>0$. By applying such a simple algebraic manipulation, it can be proved that $a_{1} a_{2}-a_{3}>0$. Hence, the endemic equilibrium point is local asymptotically stable.

\section{Parameter Estimation}

There are eight parameters in the SIRS model (2). In this paper, the three parameters are fixed, and the other five parameters are estimated. The idea of the estimation parameter is to minimize the objective function (1) so that the parameter values correspond to the real data. The first step to estimate the parameter is to define the initial values $\vec{\theta}_{0}=(\alpha, \gamma, v, \delta, \beta)=(0.000010260,1.4$, $0.3816,0.982,0.1667)$. In the first period January 2015, the total population in Malang is 866801 , so we choose the initial values of the population as $\left(S_{0}, I_{0}, R_{0}\right)=(866774,27,0)$. By following step 1 until step 9 in the Nelder Mead algorithm, which was described in the previous section, the iteration ends after the error tolerance is met. The parameter that makes the minimum objective function is the best one. The minimum values of the objective function was reached in the $1412^{\text {th }}$ iteration. The best parameter values were presented at Table 2 , and they can be used to determine the value of the basic reproduction number, namely $\mathcal{R}_{0}=$ 0.00000167

Table 2. Estimated parameter value

\begin{tabular}{ccc}
\hline Parameter & Value & Source \\
\hline$\Lambda$ & 0.0025128 & Dispendukcapil Malang \\
$\alpha$ & 0.0000311 & Estimated \\
$\gamma$ & 0.022 & Estimated \\
$\mu_{0}$ & 0.0024205 & Dispendukcapil Malang \\
$v$ & 0.965 & Estimated \\
$\delta$ & 1.788 & Estimated \\
$\mu_{1}$ & 0.0000018 & RSUD Dr. Saiful Anwar \\
$\beta$ & 1.248 & Estimated \\
\hline
\end{tabular}

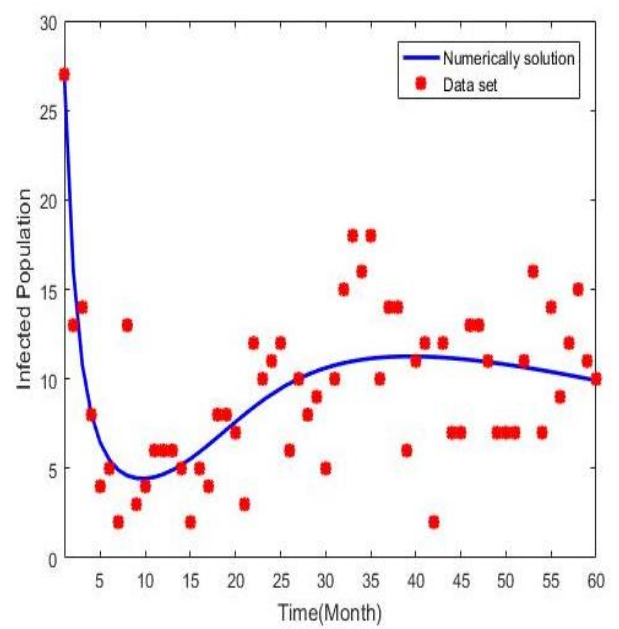

Figure 1. Comparison of SIRS model of Hepatitis B and the infected data

\section{Numerical Simulation}

The SIRS model (2) is simulated by using the best parameter presented at Table 2 . Figure 1 shows that the numerical solution of the model approach the trend of the infected data of Hepatitis B.

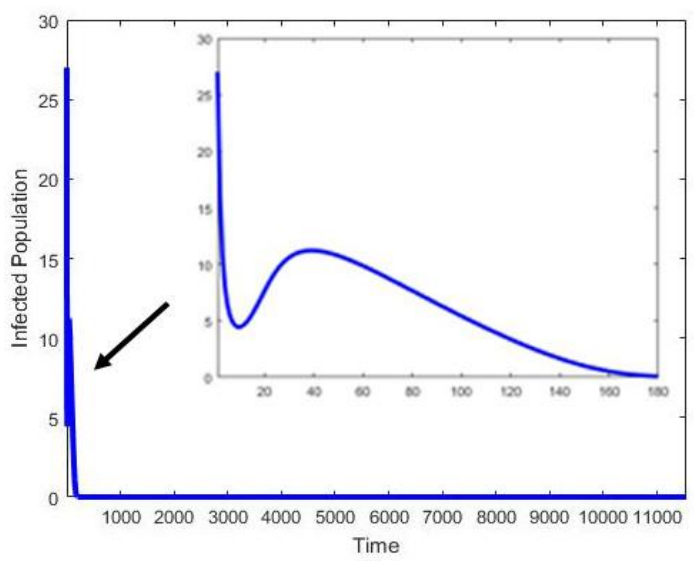

Figure 2. Simulation SIRS model of Hepatitis B 
Figure 2 shows the profile of the density of the infected population when the length of the interval time is enlarged becomes $t=11544$. In accordance with the analytical result, eventually, the size of the infected population decreases and converges to 0 because the basic reproduction number $\mathcal{R}_{0}<1$.

\section{CONCLUSION}

A SIRS model representing the spread of the Hepatitis B disease model has been reconstructed in the form of a three-dimensional nonlinear autonomous differential equation system with eight parameters. The performed dynamical analysis shows that the model has a disease-free equilibrium point and an endemic equilibrium point. By applying the Next Generation Matrix method, the basic reproduction number $\mathcal{R}_{0}$ has been formulated. The existence and the stability of the equilibrium points depend on the basic reproduction number $\mathcal{R}_{0}$. The disease-free point always exists, while the endemic point only exists when $\mathcal{R}_{0}>1$. By applying linearization around each equilibrium point, the local stability of the equilibrium points is investigated. The disease-free point is local asymptotically stable when $\mathcal{R}_{0}<1$. Referring to the Routh-Hurwitz criteria, it can be shown that the endemic point is local asymptotically stable when this point exists.

Based on the data of infected cases taken from a hospital in Malang, Indonesia, the five parameters of the model have been estimated. The numerical simulation of the SIRS model by using the estimated parameters reach the minimum error if compared to the infected data. The result of the simulation of the proposed model predicts that eventually, Malang can be a Hepatitis B-disease-free state. This result agrees with the analytical result since the estimated parameters give $\mathcal{R}_{0}<1$.

\section{REFERENCES}

[1] Wong, D.J., S.A. Locarnini. 2018. Molecular virology and life cycle. In: Kao, J.H., D.S. Chen (Eds). Hepatitis B Virus and Liver Disease. Springer. Singapore. 1-23.

[2] Konerman, M.A., A.S. Lok. 2018. Epidemiology, diagnosis, and natural history of Hepatitis B. In: Sanyal, A.J., T.D. Boyer, K.D. Lindor, N. A. Terrault. Zakim and Boyer's Hepatology: A Textbook of Liver Disease, $7^{\text {th }}$ Ed. Elsevier. 474-484.

[3] Muljono, D.H. 2017. Epidemiology of Hepatitis B and C in Republic of Indonesia.
Euroasian. J. Hepatogastroenterol. 7(1). 55-

59.

[4] Kermack, W.O., McKendrick A.G. 1927. A Contribution to the mathematical theory of epidemics. Proceedings of the Royal Society of London. Series A. 115(772). 700-721.

[5] Capasso, V., G. Serio. 1978. A generalization of the Kermack-McKendrick deterministic epidemic model. Math.I Biosci. 42(1-2). 43-61.

[6] Khan, T., Z. Ullah, N. Ali, G. Zaman. 2019. Modeling and control of the Hepatitis B virus spreading using an epidemic model. Chaos Solitons Fractals. 124. 1-9.

[7] Golgeli, M. 2019. A mathematical model of Hepatitis B transmission in Turkey. Communication Faculty of Sciences, University of Ankara Series A1 Mathematics and Statistics. 68. 1586-1595.

[8] Wolberg, J. 2006. Data Analysis using the Method of Least Squares: extracting the most information from experiments. SpringerVerlag. Berlin Heidelberg. Germany.

[9] Lagarias, J.C., J.A. Reeds, M.H. Wright, P.E. Wright. 1998. Convergence properties of the Nelder-Mead Simplex Method in low dimensions. Soc. Ind. Appl. Math. 9. 112-147.

[10] Gao, F., L. Han. 2010. Implementing the Nelder-Mead Simplex algorithm with adaptive parameters. Comput. Optim. Appl. 51. 259-277.

[11] Isermann, R., M. Munchhof. 2011. Identification of dynamic systems: an introduction with applications. Springer-Verlag. London, New York.

[12] Brauer, F., C.C. Chavez. 2012. Mathematical models in population biology and epidemiology, $2^{\text {nd }}$ Ed. Springer-Verlag. New York.

[13] Murray, J.D. 2002. Mathematical biology I. An introduction, $3^{\text {rd }}$ Ed. Springer-Verlag. Berlin Heidelberg. 